\title{
Equivalence Relations of Permutations Generated by Constrained Transpositions
}

\author{
Stephen Linton $1 \llbracket$ and James Propp $\|^{2}$ and Tom Roby ${ }^{3}$ and Julian West $\|^{\prime}$
}

${ }^{1}$ Centre for Interdisciplinary Research in Computational Algebra,

University of St Andrews, St Andrews, Fife KY16 9SX, Scotland

${ }^{2}$ University of Massachusetts, Lowell, MA 01854, USA

${ }^{3}$ University of Connecticut, Storrs, CT 06269, USA

${ }^{4}$ University of Bristol, Bristol BS8 1TW, England

\begin{abstract}
We consider a large family of equivalence relations on permutations in $S_{n}$ that generalise those discovered by Knuth in his study of the Robinson-Schensted correspondence. In our most general setting, two permutations are equivalent if one can be obtained from the other by a sequence of pattern-replacing moves of prescribed form; however, we limit our focus to patterns where two elements are transposed, conditional upon the presence of a third element of suitable value and location. For some relations of this type, we compute the number of equivalence classes, determine how many $n$-permutations are equivalent to the identity permutation, or characterise this equivalence class. Although our results include familiar integer sequences (e.g., Catalan, Fibonacci, and Tribonacci numbers) and special classes of permutations (layered, connected, and 123-avoiding), some of the sequences that arise appear to be new.

Résumé. Nous considérons une famille de relations d'equivalence sur l'ensemble $S_{n}$ des permutations, qui généralisent les relations de Knuth liées à la correspondance Robinson-Schensted. Dans notre contexte général, deux permutations sont considérées comme équivalentes si l'une peut être obtenue de l'autre auprès d'une séquence de remplacements d'un motif par un autre selon des règles précisées. Désormais, nous ne considérons dans l'oeuvre actuelle que les motifs qui correspondent à la transposition de deux éléments, conditioné sur la presence d'un élément de valeur et de position approprié. Pour plusieurs exemples de ce problème, nous énumérons les classes d'équivalence, nous déterminons combien de permutations sur $n$ éléments sont équivalentes à l'identité, ou nous précisons la forme des éléments dans cette dernière classe. Bien que nos résultats retrouvent des séquences des entiers très bien connues (nombres de Catalan, de Fibonacci, de Tribonacci ...) ainsi que des classes de permutations déjà étudiées (en couches, connexes, sans motif 123), nous trouvons également des séquences qui paraissent être nouvelles.
\end{abstract}

Keywords: permutation patterns, equivalence classes, Knuth relations, integer sequences, Catalan numbers, layered permutations, connected permutations, pattern-avoiding permutations

\footnotetext{
${ }^{\dagger}$ Supported by EPSRC grant EP/C523229/01.

¥Supported by NSF

$\S$ Supported by NSERC operating grant OGP0105492.

1365-8050 @ 2010 Discrete Mathematics and Theoretical Computer Science (DMTCS), Nancy, France
} 


\section{Introduction and motivation}

We consider a family of equivalence relations on permutations in $S_{n}$, in which two permutations are considered to be equivalent if one can be converted into the other by replacing a short subsequence of elements by the same elements permuted in a specific fashion, or (extending by transitivity) by a sequence of such moves. These generalise the relations discovered by Knuth in his study of the Robinson-Schensted correspondence, though our original motivations were unrelated. We begin the systematic study of such equivalence relations, connecting them with integer sequences both familiar and (apparently) new.

As a simple first example, the permutation 123456 can be converted to 125436 by replacing the subsequence 345 by $\mathbf{5 4 3}$. The same permutation 123456 can be also be converted into 163452 . In each of these examples, the subsequence removed is of pattern 123 (reducing the elements to form a permutation whose elements are in the same relative order) and the subsequence replacing it is of pattern 321.

We could therefore say that 123456 and 125436 are equivalent under the replacement $123 \rightarrow 321$. Since we want all our replacement rules to be bi-directional, we will actually say that these permutations are equivalent under $123 \leftrightarrow 321$, or, using set notation, under $\{123,321\}$. Since 163452 and 123456 are equivalent under the same replacement, by transitivity we also have that 163452 and 125436 are equivalent under $\{123,321\}$.

Interesting enumerative questions arise when the elements being replaced are allowed to be in general position (Section 2), but also when the replacements are further constrained to affect only adjacent elements as in the very first example above (Section 3), or even when constrained to affect only blocks of consecutive elements representing a run of consecutive values, again as in the first example (Section 4 .

We may also wish to allow more than one type of (bi-directional) replacement, such as both $123 \leftrightarrow 321$ and $123 \leftrightarrow 132$. If the intersection of these sets is nonempty, the new relation corresponds simply to a union of the two sets: $\{123,132,321\}=\{123,321\} \cup\{123,132\}$. However, the Knuth relations (described below) require two disjoint types of replacements.

Let $\pi \in S_{n}$, and let $P=\left\{B_{1}, B_{2}, \ldots, B_{t}\right\}$ be a (set) partition of $S_{k}$, where $k \leq n$. Each block $B_{l}$ of $P$ represents a list of $k$-length patterns which can replace one another. We call two permutations $P^{\circ}$-equivalent if one can be obtained from the other by a sequence of replacements, each replacing a $\sigma_{i}$-pattern subsequence with the same elements in the pattern $\sigma_{j}$, where $\sigma_{i}$ and $\sigma_{j}$ lie in the same block $B_{l}$ of $P$. Then $\mathrm{Eq}^{\circ}(\pi, P)$ will denote the set of permutations equivalent to $\pi$ under $P^{\circ}$-equivalence. Thus $163452 \in \mathrm{Eq}^{\circ}(123456,\{\{123,321\}\})$.

Similarly, we will use $P^{\prime \prime}$ to denote the equivalence relation and $\mathrm{Eq} "(\pi, P)$ for the equivalence class of $\pi$ under replacement within $P$ only of adjacent elements, e.g. $125436 \in \mathrm{Eq}^{\prime \prime}(123456,\{\{123,321\}\})$. And we will use $\mathrm{Eq}^{\square}(\pi, P)$ for the case where both positions and values are constrained, e.g. $125436 \in$ $\mathrm{Eq}^{\square}(123456,\{\{123,321\}\})$. To refer to such classes generally we use the notation $\mathrm{Eq}^{\star}(\pi, P)$. The set of distinct equivalence classes into which $S_{n}$ splits under an equivalence $P^{\star}$ will be denoted $\operatorname{Classes}^{\star}(n, P)$.

The present paper begins the study of these equivalence relations by considering three types of question:

(A) Compute the number of equivalence classes, \# Classes $^{\star}(n, P)$, into which $S_{n}$ is partitioned.

(B) Compute the size, $\# \mathrm{Eq}^{\star}\left(\iota_{n}, P\right)$, of the equivalence class containing the identity $\iota_{n}=123 \cdots n$.

(C) (More generally) characterise the set $\mathrm{Eq}^{\star}\left(\iota_{n}, P\right)$ of permutations equivalent to the identity.

Although the framework above allows for much greater generality, in this paper we will restrict our attention to replacements by patterns of length $k=3$, and usually to replacement patterns built up from pairs in which one permutation is the identity, and the other is a transposition (i.e., fixes one of the elements). Omitting some cases by symmetry, we have the following possible partitions of $S_{3}$, where (as 
usual) we omit singleton blocks:

$$
\begin{aligned}
& P_{1}=\{\{123,132\}\}, \\
& P_{2}=\{\{123,213\}\}, \\
& P_{4}=\{\{123,321\}\} .
\end{aligned}
$$

We will also consider applying two of these replacement operations simultaneously, and we will number the appropriate partitions as

$$
\begin{aligned}
& P_{3}=\{\{123,132,213\}\}, \\
& P_{5}=\{\{123,132,321\}\}, \\
& P_{6}=\{\{123,213,321\}\},
\end{aligned}
$$

following the convention $P_{i+j}:=P_{i} \vee P_{j}$, the join of these two partitions [EC1, ch.3]. Indeed we can allow all three replacements: $P_{7}=\{\{123,132,213,321\}\}$. (In fact, the cases $P_{1}$ and $P_{2}$ are equivalent by symmetry, as are $P_{5}$ and $P_{6}$. We list $P_{1}$ and $P_{2}$ separately so as to be able to consider their join.)

Our motivation for focussing attention on pairs of this form is that we can then think of an operation, not in terms of replacing one pattern by another, but simply in terms of swapping two elements within the pattern, with the third serving as a witness enabling the swap.

By far the best-known example of constrained swapping in permutations is certainly the Knuth Relations [Knu70], which allow the swap of adjacent entries provided an intermediate value lies immediately to the right or left. In the notation of this paper, they correspond to $P_{K}^{\|}=\{\{213,231\},\{132,312\}\}$. Permutations equivalent under this relation map to the same first coordinate ( $P$-tableau) under the RobinsonSchensted correspondence.

Mark Haiman introduced the notion of dual equivalence of permutations: $\pi$ and $\tau$ are dual equivalent if one can be obtained from the other by swaps of adjacent values from the above $P_{K}$, i.e., if their inverses are Knuth-equivalent, or if they map to the same second coordinate ( $Q$-tableau) under the RobinsonSchensted correspondence [Hai92]. For the enumerative problems in this paper, we get the same answers for Knuth and dual equivalence.

In her dissertation [SA07] Sami Assaf constructed graphs (with some extra structure) whose vertices are tableaux of a fixed shape (which may be viewed as permutations via their "reading words"), and whose edges represent (elementary) dual equivalences between vertices. For this particular relation (equivalently for the Knuth relations), she was able to characterise the local structure of these graphs, which she later used to give a combinatorial formula for the Schur expansion of LLT polynomials and MacDonald Polynomials. She also used these, along with crystal graphs, to give a combinatorial realization of Schur-Weyl duality [SA08].

S. Fomin has a very clear elementary exposition of how Knuth and dual equivalence are related to the Robinson-Schensted correspondence, Schützenberger's jeu de taquin, and the Littlewood-Richardson rule in [EC2, Ch. 7, App. 1]. For the problems considered above, the answers for $P_{K}^{\prime \prime}$ are well known to be: (A) the number of involutions in $S_{n}$; (B) 1 ; and (C) $\{i d\}$. In fact one can compute \#Eq" $\left(\pi, P_{K}\right)$ for any permutation $\pi$ by using the Frame-Robinson-Thrall hook-length formula to compute the number of standard Young tableaux of the shape output by the R-S correspondence applied to $\pi$.

Given that the Knuth relations act on adjacent elements, and lead to some deep combinatorial results, it is perhaps not surprising that the most interesting problems and proofs in this paper are to be found in Section 3 A summary of our numbers and results is given in Figure 1 . 


\section{Fig. 1: Summary of Results}

These tables give numerical values and names (when available) of the sequences associated with our two main enumerative questions. All sequences begin with the value for $n=3$. Results proven in this paper have a grey background; for other cases we lack even conjectural formulae. Six-digit codes preceded by "A" cite specific sequences in Sloane [OEIS].

\begin{tabular}{|c|c|c|c|c|}
\hline \multicolumn{2}{|c|}{ Number of classes } & $\begin{array}{l}\S 1 \\
\text { neither ("classical") }\end{array}$ & $\begin{array}{l}\S 2 \\
\text { only indices adjacent }\end{array}$ & $\begin{array}{l}\S 3 \\
\text { indices and values adjacent }\end{array}$ \\
\hline (1) & $123 \leftrightarrow 132$ & {$[5,14,42,132,429]$} & $\begin{array}{lllllll}5 & 16 & 62 & 284 & 1507 & 0104\end{array}$ & $\begin{array}{lllllll}5 & 20 & 102 & 626 & 4458 & 36144\end{array}$ \\
\hline (2) & $123 \leftrightarrow 213$ & Catalan & {$[5,10,62,284,150 /, 9104]$} & {$[3,20,102,020,4458,30144]$} \\
\hline (4) & $123 \leftrightarrow 321$ & $\begin{array}{l}{[5,10,3,1,1,1]} \\
\text { trivial }\end{array}$ & {$[5,16,60,260,1260,67442]$} & {$[5,20,102,626,4458,36144]$} \\
\hline (3) & $123 \leftrightarrow 132 \leftrightarrow 213$ & $\begin{array}{l}{[4,8,16,32,64,128]} \\
\text { powers of } 2\end{array}$ & $\begin{array}{l}{[4,10,26,76,232,764]} \\
\text { involutions }\end{array}$ & {$[4,17,89,556,4011,32843]$} \\
\hline (5) & $123 \leftrightarrow 132 \leftrightarrow 321$ & {$[4,2,1,1,1,1]$} & {$\left[\begin{array}{lllllll}4 & 8 & 14 & 27 & 68 & 159 & 496\end{array}\right]$} & {$\left[\begin{array}{lllllll}4 & 16 & 84 & 536 & 3912 & 32256\end{array}\right]$} \\
\hline (6) & $123 \leftrightarrow 213 \leftrightarrow 321$ & trivial & & \\
\hline (7) & $\begin{array}{l}123 \leftrightarrow 132 \\
\leftrightarrow 213 \leftrightarrow 321\end{array}$ & $\begin{array}{l}{[3,2,1,1,1,1]} \\
\text { trivial }\end{array}$ & {$[3,4,5,8,11,20,29,57]$} & {$[3,13,71,470,3497]$} \\
\hline
\end{tabular}

\begin{tabular}{|c|c|c|c|c|}
\hline \multicolumn{2}{|c|}{ Size of class with $\iota_{n}$} & $\begin{array}{l}\S 1 \\
\text { neither ("classical") }\end{array}$ & $\begin{array}{l}\S 2 \\
\text { only indices adjacent }\end{array}$ & $\begin{array}{l}\S 3 \\
\text { indices and values adjacent }\end{array}$ \\
\hline (1) & $123 \leftrightarrow 132$ & {$[2,6,24,120,720]$} & {$[2,4,12,36,144,576,2880]$} & {$[2,3,5,8,13,21,34,55]$} \\
\hline (2) & $123 \leftrightarrow 213$ & $(n-1) !$ & product of two factorials & Fibonacci numbers \\
\hline (4) & $123 \leftrightarrow 321$ & $\begin{array}{l}{[2,4,24,720]} \\
\text { trivial }\end{array}$ & $\begin{array}{l}{[2,3,6,10,20,35,70,126]} \\
\text { central binomial coefficients }\end{array}$ & $\begin{array}{l}{[2,3,4,6,9,13,19,28]} \\
\text { A000930 }\end{array}$ \\
\hline (3) & $123 \leftrightarrow 132 \leftrightarrow 213$ & $\begin{array}{l}{[3,13,71,461]} \\
\text { connected A003319 }\end{array}$ & $\begin{array}{l}{[3,7,35,135,945,5193]} \\
\text { terms are always odd }\end{array}$ & $\begin{array}{l}{[3,4,8,12,21,33,55,88]} \\
\text { A052952 }\end{array}$ \\
\hline (5) & $123 \leftrightarrow 132 \leftrightarrow 321$ & {$[3,23,120,720]$} & {$[3,9,54,285,2160,15825]$} & {$[3,5,9,17,31,57,105,193]$} \\
\hline (6) & $123 \leftrightarrow 213 \leftrightarrow 321$ & trivial & separate formulae for odd/even & tribonacci numbers A000213 \\
\hline (7) & $\begin{array}{l}123 \leftrightarrow 132 \\
\leftrightarrow 213 \leftrightarrow 321\end{array}$ & $\begin{array}{l}{[3,23,120,720]} \\
\text { trivial }\end{array}$ & {$[4,21,116,713,5030]$} & $\begin{array}{l}{[4,6,13,23,44,80,149,273]} \\
\text { tribonacci A000073 - }[n \text { even }]\end{array}$ \\
\hline
\end{tabular}

If $\tau \in \operatorname{Eq}^{\star}(\pi, P)$ we will say that $\tau$ is reachable from $\pi$ (under $P$ ). If $\operatorname{Eq}^{\star}\left(\iota_{n}, P\right)=S_{n}$, then every permutation in $S_{n}$ is reachable from every other, and we will say that $S_{n}$ is connected by $P$. If $\mathrm{Eq}^{\star}(\pi, P)=\{\pi\}$ we will say that $\pi$ is isolated (under $P$ ).

It is obvious that if $P_{i}$ refines $P_{j}$ as partitions of $S_{k}$ (i.e., $P_{i} \leq P_{j}$ in the lattice of partitions of $S_{k}$ ), then the partition of $S_{n}$ induced by $P_{i}$ refines the one induced by $P_{j}$, because a permutation reachable from $\pi$ under $P_{i}$ is also reachable under $P_{j}$. This enables the following simple observations:

Proposition 1 If $P_{i}$ refines $P_{j}$ (as partitions of $S_{k}$ ), then for all $\pi \in S_{n}$ with $n \geq k$

$$
\begin{array}{r}
\mathrm{Eq}^{\star}\left(\pi, P_{i}\right) \subseteq \mathrm{Eq}^{\star}\left(\pi, P_{j}\right) \\
\# \mathrm{Eq}^{\star}\left(\pi, P_{i}\right) \leq \# \mathrm{Eq}^{\star}\left(\pi, P_{j}\right) \\
\# \operatorname{Classes}^{\star}\left(n, P_{i}\right) \geq \# \operatorname{Classes}\left(n, P_{j}\right)
\end{array}
$$




\section{General pattern equivalence}

Many of the equivalence relations in this section are trivial, and follow immediately from the following observation. The others lead to familar combinatorial numbers and objects.

Proposition 2 Let $P$ be any partition of $S_{k}$ for $2 \leq k \leq n-1$. If $\# \operatorname{Classes}^{\circ}(n-1, P)=1$, then $\# \operatorname{Classes}^{\circ}(n, P)=1$.

Proof: We will show that any $\pi \in S_{n}$ can be reached from the identity, under the supposition that any two permutations in $S_{n-1}$ are equivalent. If $\pi(1) \neq n$, simply apply the supposition to the elements $1 \ldots n-1$ to obtain any permutation beginning with $\pi(1)$. Then apply the supposition to the elements now occupying positions $2 \ldots n$ to complete the construction of $\pi$.

If $\pi(1)=n$, it is necessary to add an additional step at the beginning in order to detach 1 from the tail of the permutation and move it into (say) position $n-1$. But we know we can do this by applying the supposition to the elements in positions $2 \ldots n$. And as long as $n-1 \geq 2$, position $n-1$ is among the positions $2 \ldots n$.

The following results follow.

Proposition $3{\text { \# } \operatorname{Classes}^{\circ}(n,\{\{123,132,321\}\})=1 \text { and \#Classes }}^{\circ}(n,\{\{123,132,213,321\}\})=1$ for $n \geq 5$; and \# Classes $^{\circ}(n,\{\{123,321\}\})=1$ for $n \geq 6$.

Proof: It is easy to verify by hand, or by computer, that all permutations in $S_{5}$ are reachable from 12345 by moves in $P_{5}=\{\{123,132,321\}\}$. (Indeed, all permutations in $S_{4}$ are reachable from 1234 except for 3412 , which is isolated.) As $S_{5}$ is connected, it follows (by induction) from the preceding proposition that $S_{n}$ is connected for all $n \geq 5$. Since $P_{7} \geq P_{5}$, Proposition 1 tells us that $S_{n}$ is connected under $P_{7}=\{\{123,132,213,321\}\}$ whenever it is connected under $P_{5}$. (In $S_{4}$, the permutation 3412 remains isolated.) Finally, we can check by computer that under $P_{4}=\{\{123,321\}\} S_{6}$ is connected; whence, $S_{n}$ is connected for $n \geq 6$.

We remark that under $P_{4}, S_{4}$ splits into 10 equivalence classes, and $S_{5}$ into three classes. The class containing 12345 contains 24 elements. This suggests a possible bar bet. Hand your mark six cards numbered 1 through 6 and invite him or her to lay them out in any sequence. By applying moves of the form $123 \leftrightarrow 321$ ("interchange two cards if and only if an intermediate (value) card intervenes") you will always be able to put the cards in order (although it may take some practice to become efficient at this!). Now "go easy" on your mark by reducing the number of cards to 5. Even from a random sequence, the mark has only one chance in five of being able to reach the identity.

Of course from Proposition 3 it immediately follows that:

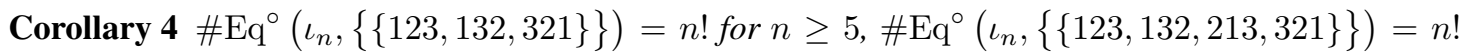
for $n \geq 5$, and $\# \mathrm{Eq}^{\circ}\left(\iota_{n},\{\{123,321\}\}\right)=n$ ! for $n \geq 6$.

Proposition $5 \mathrm{ZEq}^{\circ}\left(\iota_{n},\{\{123,213\}\}\right)=(n-1)$ ! for $n \geq 2$.

Proof: Obviously the largest element $n$ cannot be moved away from the end of the permutation. Equally obviously the $n$, remaining at the far right, facilitates the free permutation of all other elements.

Proposition 6 For $n \geq 1$, \# $\operatorname{Classes}^{\circ}(n,\{\{123,213\}\})=c_{n}=\frac{2 n !}{n !(n+1) !}$, the nth Catalan number. 
Proof: (Sketch) If $i<j<k$, and $p(i)<p(j)<p(k)$, then $p(k)$ facilitates the swapping of $p(i)$ and $p(j)$ to arrive at a permutation with a strictly larger number of inversions.

Thus the "largest" (by number of inversions) elements in each equivalence class are exactly the 123avoiding permutations, of which there are $c_{n}$ [Bon06, ch. 14] or [Bon04, Sec. 4.2]. Likewise the "smallest" elements are the 213-avoiding permutations.

The next two propositions study a class equivalent under symmetry (complementation) to $\mathrm{Eq}^{\circ}\left(\iota_{n}, P_{3}\right)$. The first references the indecomposable permutations [OEIS, A003319] or [Bon04, p. 145], and the second the layered permutations introduced by W. Stromquist [Stro93], and studied carefully by A. Price in his thesis [Pri97].

Proposition 7 Let $\rho_{n}$ denote the permutation $n, n-1, \ldots, 1$. Then $\mathrm{Eq}^{\circ}\left(\rho_{n},\{\{321,312,231\}\}\right)$ is the set of indecomposable permutations.

Proof: When viewed as a matrix, any permutation decomposes into irreducible blocks along the main diagonal. The identity $\iota_{n}$ decomposes into $n$ singleton blocks, while $\rho_{n}$ is indecomposable and is one large block.

First note that if a transformation $\left(a_{1}, a_{2}, a_{3}\right) \rightarrow\left(b_{1}, b_{2}, b_{3}\right)$ applied within a block causes it to split into more than one block, then $b_{1}$ must be in the leftmost/lowest of the new blocks, and $b_{3}$ in the rightmost/highest. Therefore $b_{1}$ must be less than $b_{3}$, which is exactly what doesn't happen with any of our possible transformations, because the first element is larger than the third in each of 321, 312 and 231. Thus, in particular, if we start with an indecomposable permutation such as $\rho_{n}$, successive applications of the permitted operations will always produce indecomposable permutations.

Next, we have to show that all indecomposable permutations are in fact reachable from $\rho_{n}$. Remembering that our replacement operations are all reversible, we will instead show that we can always return to $\rho_{n}$ from an arbitrary indecomposable permutation.

Take $n \geq 3$, and let $\tau=t_{1}, t_{2}, \ldots, t_{n}$ be an arbitrary indecomposable permutation other than $\rho_{n}$. We will show that $\tau$ always contains at least one of 312 or 231 .

If we are not in $\rho_{n}$ then somewhere there is $i<j$ such that $t_{i}<t_{j}$. It can't be the case that $t_{i}>t_{i+1}>$ $\ldots>t_{j-1}>t_{j}$, so somewhere there is a consecutive rise, say $t_{k}, t_{k+1}$. Now if any element to the right of $t_{k+1}$ is less than $t_{k}$ we have a 231, so assume there are none such. Similarly, assume there is no element to the left of $t_{k}$ and greater than $t_{k+1}$.

But there must be some $x$ to the left of $t_{k}$ which is greater than some $y$ to the right of $t_{k+1}$, or otherwise the permutation decomposes between $t_{k}$ and $t_{k+1}$. These four elements $x, t_{k}, t_{k+1}, y$ form a 3142 , which contains both a $312\left(x, t_{k}, y\right)$ and a $231\left(x, t_{k+1}, y\right)$.

Having now located a 312 or 231 , we can then apply either $312 \rightarrow 321$ or $231 \rightarrow 321$, as appropriate. Each of these operations simply switches a pair of elements, and (as we have seen in the proof of Proposition 6) strictly increases the number of inversions, progressing us toward $\rho_{n}$. This completes the proof that all indecomposable permutations are reachable, and therefore the proof that the reachable permutations are exactly the indecomposable permutations.

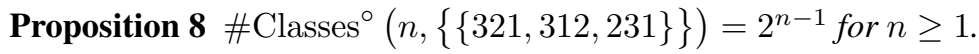

Proof: This follows fairly easily from comments made in the proof of the previous proposition. We found that the equivalence class of the anti-identity consisted of the indecomposable permutations. 
Any permutation decomposes as a direct sum of irreducible blocks. We found that in our case, our operations cannot cause a block to split. Therefore they also cannot cause blocks to join up, because then the operation could be reversed, splitting the blocks. So the block composition is preserved.

By the arguments already given, we can work within any indecomposable block to restore it to an antiidentity. Therefore each equivalence class consists of all the permutations with a given block structure under direct sum of indecomposables.

In particular, each equivalence class contains exactly one permutation which is a direct sum of antiidentities. These are exactly the layered permutations, and there are clearly $2^{n-1}$ of them, with a factor of 2 according to whether each consecutive pair of elements is or is not in the same layer.

Finally we apply the reversal involution on $S_{n}$ to the above result to get our result for the partition $P_{3}$.

Theorem 9 \#Classes $^{\circ}(n,\{\{123,132,213\}\})=2^{n-1}$ for $n \geq 1$.

\section{Adjacent transformations}

As mentioned in the introduction, this section contains our most interesting results and proofs. The first rediscovers sequence A010551 from Sloane [OEIS].

Theorem 10 \#Eq" $\left(\iota_{n},\{\{123,213\}\}\right)=\lfloor n / 2\rfloor !\lceil n / 2\rceil$ ! for $n \geq 1$.

Proof: The largest element, $n$, never comes unglued from the end, because there is nothing to enable it. And therefore $n-1$ must stay somewhere in the last three positions (as only $n$ can enable its movement), and $n-2$ somewhere in the last five, and so on; such restrictions apply to $\lfloor n / 2\rfloor$ of the elements. This limits the number of potentially reachable elements to $\lfloor n / 2\rfloor !\lceil n / 2\rceil !$ : placing the elements from largest to smallest, one has a choice of $1,2,3, \ldots,\lfloor n / 2\rfloor,\lceil n / 2\rceil, \ldots, 3,2,1$ places to put each element.

Next we will show that all permutations conforming to these restrictions are indeed reachable. We will do this in two stages. In Stage 1 we advance each of the large, constrained elements as far left as it can go In Stage 2 we construct the target permutation from left to right, two elements at a time.

Stage 1: The elements $\lfloor n / 2\rfloor, \ldots, n-1, n$ are to be positioned. First move $\lfloor n / 2\rfloor$ one step left, using a move of type $123 \rightarrow 213$, in which $\lfloor n / 2\rfloor+1$ plays the role of the facilitating " 3 ". In just the same way, more the element $\lfloor n / 2\rfloor+1$ to the left, continuing until the entire block $\lfloor n / 2\rfloor, \ldots, n-1$ has been shifted one to the left. The element $n-1$ has now reached its leftmost permitted position, and will remain in place as we now move the block $\lfloor n / 2\rfloor, \ldots, n-2$. This moves $n-2$ as far left as it will go, and we now move the next smaller block, etc. As promised, this places each constrained element as far left as possible. These elements will now serve as a "skeleton" enabling the construction of the target permutation.

Stage 2: The key observation making this stage possible is that the small, unconstrained elements can be freely moved about, leaving the large elements in the skeleton fixed. This is because, if $\{a, b\}<X<Y$, we can always execute the following sequence of moves: $a X b Y \rightarrow a b X Y \rightarrow b a X Y \rightarrow b X a Y$. Also in the case where $n$ is odd, the leftmost element in the skeleton is in position 3 , and the two small elements in positions 1 and 2 can be interchanged if desired.

Now we examine the target permutation and move the required element(s) into the first position (if $n$ is even), or the first two positions (if $n$ is odd). At this point, the elements occupying the next two positions are reclassified as small, so that the skeleton terminates two positions further to the right, and we continue by placing and ordering the next pair of elements. By continuing two elements at a time, we can build the entire target permutation. 
Theorem 11 (a) \#Eq" $\left(\iota_{n},\{\{123,132,321\}\}\right)=\frac{3}{2}(k)(k+1)(2 k-1) !$, for $n=2 k+1$ odd and $n \geq 3$. (b)\#Eq" $\left(\iota_{n},\{\{123,132,321\}\}\right)=\frac{3}{2}(k)\left(k-\frac{1}{3}\right)(2 k-2) !-(2 k-3) !$, for $n=2 k$ even and $n \geq 2$. (Here $(2 k-3) ! !=(2 k-3) \ldots(3)(1)$, the product of odd natural numbers less than or equal to $(2 k-3)$.

Proof: (Sketch) As in the previous proof, we begin by giving a necessary set of conditions for a permutation to be reachable. We then show how to reach all such permutations, except for a small number of permutations in the even case, which we explain separately.

Observe that the element 1 cannot occupy a position of even index, and the element 2 cannot occupy a position of odd index to the left of 1 . Call this $\mathcal{A}$, the class of admissible permutations. A simple counting argument shows that this characterization produces the given formula for odd $n$, and also gives formula for even $n$ upon suppression of the double-factorial correction term. This discrepancy is because when $n=2 k$ is even there are a small number of exceptional permutations which must be excluded; we will turn to these at the end of the proof.

Continuing on the assumption that $n=2 k+1$ is odd, it remains to show that all admissible permutations are in fact reachable. We do this in two stages.

Stage 1: First we will show that all permutations beginning with a 1 are reachable from the identity. We proceed in steps; after each, we will have a monotonically increasing initial segment, followed by a completed target segment. Each step increases the length of the completed segment by 1 by selecting and moving one element. If the selected element $(b>1)$ is an even number of positions $(2 k)$ away from the place to which it is to be moved, perform $k$ times the following pair of moves: $b x y \rightarrow y x b \rightarrow x y b$; if an odd number of positions away, prepend $a b c x y \rightarrow a c b x y$, then continue as before. This means that we can travel between the identity and any permutation that begins with a 1 .

Stage 2: It remains to show that the element 1 can always be moved to the front of any admissible permutation. Actually, we only need to show that the element 1 can always be moved toward the front.

If the 1 is at the very end of the permutation, the 2 must be to its left and in a position of opposite parity. Move the 2 rightward using moves 123 or $132 \rightarrow 321$ until it is adjacent to the 1 ; the 1 can then be moved.

If the 1 is not at the very end of the permutation, then consider the run of five elements centered on the 1. (Note that the existence of this 5 -factor depends on the assumption that $n$ is odd.) There are 24 cases. Most can be handled locally, but those of the form 2-1-- require more care. Checking these cases completes the proof for odd $n$. Now, for even $n$ we have to consider which permutations have been included in the given characterization $\mathcal{A}$, but which are not in fact reachable.

Let $n=2 k$. Here is a description of a small class of exceptional permutations, $\mathcal{X}$, which are not reachable. Fill the positions in order $n-1, n, n-3, n-2, n-5, n-4 \ldots 3,4,1,2$. When filling positions of odd index, the smallest available element must be chosen; the subsequent selection of an element to place to its right is then unconstrained. None of these $(n-1) ! !$ permutations is reachable. However, most of them are also not in $\mathcal{A}$, because most have the 2 in position $n-3$; the only ones we have counted are the ones where the 2 is in position $n$, of which there are $(n-3) ! !$.

To see that none of the permutations in $\mathcal{X}$ is reachable, consider their 3 -factors. These are all 213,312 , or 231 ; therefore these permutations are isolated points, and not in the equivalence class of the identity.

Now we have to consider which permutations in $\mathcal{A}$ are not in fact reachable. The proof for odd $n$ only fails when the element 1 lies in the penultimate position $n-1$. We have already seen that the permutations belonging to $\mathcal{X} \cap \mathcal{A}$ are not reachable; we will show that all others are. Take any permutation $\pi \notin \mathcal{X}$, but with the minimal element 1 placed in position $n-1$. Checking the conditions from right to left, suppose 
all odd positions from $j$ to $n-1$ are occupied by left-to-right minima, but suppose that the smallest element situtated in positions 1 through $j-1$ is not in position $j-2$, as expected, and its value is $x$.

As before, all we need to do is show that we can move the element 1 to the left. This exploits two facts: that $x$ is the minimal element in a lefthand region, and the righthand region is alternating. First, take the element $x$ and shift it rightward, two positions at a time, until it arrives in position $j-2$ or $j-1$. In either case, $x$ now lies in a 321 which begins in an odd position. We check that we can propagate either of these odd 321s rightward until they capture the smallest element, which can then be moved. This completes the missing step in the proof for even $n$.

Theorem 12 \#Eq" $\left(\iota_{n},\{\{123,321\}\}\right)=\left(\begin{array}{c}n-1 \\ \lfloor(n-1) / 2\rfloor\end{array}\right)$.

Proof: (Sketch) The permutations in this class are direct sums of singletons and of blocks of odd size greater than one, where within each block the even elements are on the diagonal, and the odd elements form a plus-indecomposable [AAK03] 321-avoiding permutation. Let us call the set that we have just described $\mathcal{A}_{n}$. First we will show that $\mathcal{A}_{n}$ is closed under $123 \leftrightarrow 321$; since the identity is in $\mathcal{A}_{n}$ this will establish that the equivalence class of the identity is a subset of $\mathcal{A}_{n}$. Then we will show that we can return to the identity from any permutation in $\mathcal{A}_{n}$, which will establish that the two sets are identical.

Let $\pi$ be an arbitrary permutation belonging to $\mathcal{A}_{n}$. Call the non-singleton blocks of $\pi$ large; unless $\pi$ is the identity, it contains at least one large block. Note that large blocks begin and end with descents.

First show that any application of $123 \rightarrow 321$ to $\pi$ produces an element of $\mathcal{A}_{n}$. Consider the different ways that a $\pi_{i}, \pi_{i+1}, \pi_{i+2}$ of form 123 might occur within $\pi$. The cases to consider are (a) all three elements are in singleton blocks, (b) exactly two of the elements are in singleton blocks, (c) only the middle element is in a singleton block, (d) all three elements are in a single large block, (e) two consecutive elements are in a large block, but the third is not (which cannot arise). In each of these cases the replacement $123 \rightarrow 321$ winds up gluing together all the blocks which it straddles.

Now consider applications of $321 \rightarrow 123$ in a permutation $\rho \in \mathcal{A}_{n}$. Clearly, any 321 must lie within a single block, as in any two blocks, all the elements in the block to the right are larger than all the elements in the block to the left. Because the even elements within a block increase monotonically, the 321 is composed of odd, even, odd elements. It might, indeed, be the pattern resulting from a replacement $123 \rightarrow 321$ in any one of the cases (a) through (d) above; therefore by undoing it we might return to any one of these four configurations. But no matter, as each one corresponds to a permutation in $\mathcal{A}_{n}$.

Now we need to show that we can return to the identity from any permutation $\sigma$ in $\mathcal{A}_{n}$. Observe that every large block of $\sigma$ contains a 321 as a factor, because the first element of the block must lie below the diagonal and the last element must lie above it; therefore two consecutive odd elements exist with the first below and the second above the diagonal. Together with the even element (on the diagonal) which separates them, this forms a 321 . Unless $\pi$ is itself the identity, it contains a large block, and therefore a 321. By replacing this with a 123, we move to a permutation $\rho$ having strictly fewer inversions than $\sigma$. But as $\mathcal{A}_{n}$ is closed under such replacements, we may iterate this process, until we arrive at the identity.

This establishes that the reachable permutations are as described; now to enumerate them. The number of plus-indecomposable 321-avoiding permutations on $m+1$ elements is the Catalan number $\frac{1}{m+1}\left(\begin{array}{c}2 m \\ m\end{array}\right)$, so this is the number of possible blocks of size $2 m+1$. We will need the following generating functions:

$$
\begin{aligned}
& A=\frac{1}{\sqrt{1-4 x}}=1+2 x+6 x^{2}+20 x^{3}+70 x^{4}+\ldots \\
& B=\frac{\frac{1}{\sqrt{1-4 x}}-1}{2 x}=1+3 x+10 x^{2}+35 x^{3}+126 x^{4}+\ldots
\end{aligned}
$$




$$
C=\frac{1-\sqrt{1-4 x}}{2 x}=1+x+2 x^{2}+5 x^{3}+14 x^{4}+\ldots
$$

Now, a reachable permutation of even size $2 k+2$ is the direct sum of an indecomposable block of size $2 i+1(i \geq 0)$ and a reachable permutation of odd size $2(k-i)+1$. Likewise a reachable permutation of odd size $2 k+1$ is the direct sum of a block of size $2 i+1$ and a reachable permutation of even size $2(k-i)$. The theorem then follows by checking that $B=A C$ and $A=(1+x B) C$.

Theorem 13 (a) \#Classes" $(n,\{\{123,132,213\}\})=\operatorname{inv}_{n}$, the number of involutions of order $n$. (b) \# $\mathrm{Eq}^{\prime \prime}(\pi,\{\{123,132,213\}\})$ is odd for all $n$ and for each $\pi \in S_{n}$.

Proof: (Sketch) An involution is a partition of $[n]$ into 1-cycles and 2-cycles. Write each involution as a product of cycles, with the elements increasing within each 2-cycle, and with the cycles in decreasing order of largest element. Then drop the parentheses.

The resulting set $C$ of permutations covers all the classes, because each permutation $\pi$ can be reduced to an element of $C$ as follows: if $n$ is at the front of $\pi$, it must stay there. (This corresponds to having $n$ as a fixed point in the involution.) Otherwise, use $123 \rightarrow 132$ and $213 \rightarrow 132$ to push $n$ leftward into position 2, which is as far as it will go. The element which is thus pushed into position 1 is the minimal element which was to the left of $n$ to begin with. This is because this minimal element can never trade places with $n$ under the given operations, as 1 is left of 3 in all of 123, 132 and 213.

This shows that the number of classes is at most the same as the number of involutions. To show that they are the same, it remains to show that each $\pi$ can be reduced to a unique member of $C$. An equivalent statement (a') is that it is not possible to move from one member of $C$ to another.

We will prove this by induction on $n$. At the same time we will prove statement (b) of the theorem. Assume as an induction hypothesis that both statements have been demonstrated for $n-1$ and $n-2$.

If the largest element, $n$, is at the front of a permutation, then it cannot move from there, so the equivalence classes split into two kinds: special equivalence classes, in which $n$ is always at the front, and ordinary equivalence classes, in which $n$ is never at the front. The special equivalence classes for $S_{n}$ correspond upon deletion of the first elements to all the equivalence classes for $S_{n-1}$; therefore we can assert by induction the truth of both (a') and (b) as they apply to the special equivalence classes.

We turn to the ordinary equivalence classes. Consider a (directed) graph in which the vertices correspond to the permutations in $S_{n}$, and there is a blue (directed) edge from $\pi$ to $\rho$ if $\rho$ can be obtained from $\pi$ by applying $123 \rightarrow 132$, a red edge for each $213 \rightarrow 132$, and a green edge for each $123 \rightarrow 213$.

Now consider the forest of rooted trees which one obtains by taking only those red and blue edges in which the element $n$ plays the role of the " 3 ". The roots (i.e., sinks) of these trees are exactly the permutations in which the $n$ has advanced as far as possible, to position 2. Each node in this forest has either zero or two children, because if it has a blue child (obtained by travelling backwards along a blue edge) then it also has a red child, and vice versa. Because each node has either zero or two children, each rooted tree has an odd number of nodes; indeed all of its level-sums are even except the zeroth level sum, which corresponds to the root vertex, which we call the ground state. Now we will selectively glue trees together into larger components. Namely, two trees with ground states $g$ and $h$ will be combined if $g(1)=h(1)$ and if, upon deleting the first two elements the shortened permutations, $g^{\prime \prime}$ and $h^{\prime \prime}$ are equivalent, regarded (in the obvious way) as members of $S_{n-2}$.

We claim that these larger components are exactly the connected components of our directed graph. Therefore, to complete the proof we show, by examining various cases, that there are no directed edges in 
the graph which escape from one component to another, in other words that all allowable moves carry us between two permutations which have equivalent ground states.

This result is particularly interesting because the equivalence relation has the same number of classes as Knuth equivalence, yet the two relations appear to be materially different. For example, for $n=3$, the equivalence classes for $P_{K}$ have sizes $1,1,2,2$, whereas for $P_{3}=\{\{123,132,213\}\}$ the sizes are $1,1,1,3$.

\section{Doubly adjacent transformations}

For completeness, we include a brief treatment of the situation where both indices and values are simultaneously constrained to be adjacent. As the situation is highly constrained, it is perhaps not surprising that the permutations reachable from the identity are in each case easy to classify and enumerate. Since all the treatments are similar, we can wrap them up in one proposition.

As in the previous section, we have as yet no results related to the enumeration of equivalence classes. This proposition uses the Iverson bracket; $[\mathrm{P}]$ is equal to 1 if the statement $\mathrm{P}$ is true, and 0 otherwise.

Proposition $14 \mathrm{ERq}^{\square}\left(\iota_{n}, P_{1}\right)$ obeys the recurrence $a(n)=a(n-1)+a(n-2)$ with $a_{1}=a_{2}=1$. (Fibonacci numbers $F(n)$, OEIS A000045]).

$\# \mathrm{Eq}^{\square}\left(\iota_{n}, P_{4}\right)$ obeys the recurrence $a(n)=a(n-1)+a(n-3)$ with $a_{0}=0, a_{1}=a_{2}=1($ (IOEIS. A000930]).

$\# \mathrm{Eq}^{\square}\left(\iota_{n}, P_{3}\right)=F(n+1)-[n$ is even $]$.

$\# \mathrm{Eq}^{\square}\left(\iota_{n}, P_{5}\right)$ obeys the recurrence $a(n)=a(n-1)+a(n-2)+a(n-3)$ with $a(0)=a(1)=a(2)=1$ (Tribonacci numbers, $O E I S$ A000213]).

$\# \mathrm{Eq}^{\square}\left(\iota_{n}, P_{7}\right)=T(n+2)-[n$ is even], where $T(n)$ is sequence A000073 from Sloane [OEIS], obeying the recurrence $a(n)=a(n-1)+a(n-2)+a(n-3)$ with $a(0)=a(1)=0, a(2)=1$.

Proof: (Sketch) We begin by characterizing the various equivalence classes. In each case, these are subsets of the layered permutations, and indeed consist of direct sums of anti-identities of dimensions either 1,2 or 3 , as follows:

$P_{1}(123 \leftrightarrow 132)$ : direct sums of $\rho_{1}$ and $\rho_{2}$, not beginning with $\rho_{2}$

$P_{4}(123 \leftrightarrow 321)$ : direct sums of $\rho_{1}$ and $\rho_{3}$

$P_{3}(123 \leftrightarrow 132 \leftrightarrow 213)$ : direct sums of $\rho_{1}$ and $\rho_{2}$, including at least one $\rho_{1}$

$P_{5}(123 \leftrightarrow 132 \leftrightarrow 321)$ : direct sums of $\rho_{1}, \rho_{2}$ and $\rho_{3}$, not beginning with $\rho_{2}$

$P_{7}(123 \leftrightarrow 132 \leftrightarrow 213 \leftrightarrow 321)$ : direct sums of $\rho_{1}, \rho_{2}, \rho_{3}$, at least one of odd dimension

In each case it is easy to see that the given class remains closed under application of the appropriate operations. It is also easy in general to see how to reach a given target, especially if we cast the block sizes in the language of regular expressions. In the following, the notation $\{x y\}$ means a single block of size either $x$ or $y$. An asterisk following a number means zero or more copies of that number. An asterisk following a string in [] means zero or more copies of that string.

$P_{1}$ : The block sizes are $\left[12^{*}\right]^{*}$. Build each $12 *$ from right to left.

$P_{4}$ : The block sizes are $\{13\} *$. Build each block freely.

$P_{3}$ : Split each run of 2 s freely to get $[12 *][2 * 12 *] *[2 * 1]$. Build each block from the edges to the 1 .

$P_{5}$ : The block sizes are $\left[\{13\} 2^{*}\right]^{*}$. First use $123 \rightarrow 132$ to build all the runs of 2 from right to left. Then use $123 \rightarrow 321$ to place $3 \mathrm{~s}$.

$P_{7}$ : Build the $2 \mathrm{~s}$ first, as in the case of $P_{3}$, and then place the $3 \mathrm{~s}$. 
One now verifies all the necessary base cases, as trivially $a_{1}=1, a_{2}=1$, and $a_{3}=$ the size of the non-singleton block of $P_{j}$.

As for the recurrences, for $n>3$ :

$P_{1}: a_{n}=a_{n-1}+a_{n-2}$, by appending respectively a $\rho_{1}$ or a $\rho_{2}$

$P_{2}: a_{n}=a_{n-1}+a_{n-3}$, by appending respectively a $\rho_{1}$ or a $\rho_{3}$

$P_{5}: a_{n}=a_{n-1}+a_{n-2}+a_{n-3}$, by appending $\rho_{1}, \rho_{2}$ or $\rho_{3}$

$P_{3}$ : Count all direct sums of $\rho_{1}$ and $\rho_{2}$ (obviously Fibonacci) and then subtract 1 from the even terms to remove the special case $2 *$.

$P_{7}$ : Count all direct sums of $\rho_{1}, \rho_{2}, \rho_{3}$ to get A000073, and subtract 1 from the even terms because $2^{*}$ is disallowed. Alternatively, verify the recurrence $a_{n}=a_{n-2}+U_{n}$, where $U_{n}$ is A000213, by noting that a permutation in $\mathrm{Eq}^{\square}\left(\iota_{n}, P_{7}\right)$ is either a $\rho_{2}$ prepended to a permutation in $\mathrm{Eq}^{\square}\left(\iota_{n-2}, P_{7}\right)$, or else belongs to $\mathrm{Eq}^{\square}\left(\iota_{n-2}, P_{5}\right)$.

\section{References}

[AAK03] M. H. Albert, M. D. AtKinson, AND M. KLAZAR, The enumeration of simple permutations, J. Integer Sequences, 6, Art. 03.4.4, (2003).

[SA08] S. ASSAF, A combinatorial realization of Schur-Weyl duality via crystal graphs and dual equivalence graphs, FPSAC 2008, pp. 141-152, Discrete Math. Theor. Comput. Sci. Proc., Nancy, France, 2008.

[SA07] S. ASSAF, Dual equivalence graphs, ribbon tableaux and Macdonald polynomials, Ph.D. Thesis, UC Berkeley, 2007

[Bon04] M. Bona, Combinatorics of Permutations, Chapman \& Hall/CRC, 2004.

[Bon06] M. Bona, A Walk Through Combinatorics, 2nd Ed., World Scientific Publishing Co., 2006.

[Hai92] M. Haiman, Dual equivalence with applications, including a conjecture of Proctor, Discrete Math., 99 (1992), pp. 79-113.

[Knu70] D. Knuth, Permutations, matrices and generalized Young tableaux, Pacific J. Math., 34 (1970), pp. 709-727.

[OEIS] N.J.A. Sloane, The On-line Encyclopedia of Integer Sequences, available at http://www.research.att.com/ njas/sequences/.

[Pri97] A. L. PRICE, Packing Densities of Layered Patterns, Ph.D. Thesis, University of Pennsylvania, 1997.

[EC1] R. STANley, Enumerative Combinatorics Volume 1, no. 49 in Cambridge Studies in Advanced Mathematics, Cambridge University Press, 1999.

[EC2] R. StAnley, Enumerative Combinatorics Volume 2, no. 62 in Cambridge Studies in Advanced Mathematics, Cambridge University Press, 1999. With appendix 1 by Sergey Fomin.

[Stro93] W. Stromquist, Packing Layered Posets Into Posets, preprint, 1993, available at http://walterstromquist.com/. 